The process is easy - proposals can be as brief as two pages - and decisions are fast: it took the NSF just five days to award the first three RAPID grants after the Chilean earthquake in February 2010. The NSF also works with agencies such as NOAA and the EPA to avoid duplication of effort, to team up research groups that might work well together and, for marine disasters, to ensure available ship time.

In the Gulf, however, these coordination mechanisms don't seem to be working well enough (see page 404). Basic information about the chemical composition of the leaking oil has been slow in getting out to researchers. And, delayed by initial hopes that the spill would be capped quickly, the NSF's first RAPID grants are only now being awarded, a month after the crisis began. NOAA has been slow to respond to public concerns about its ability to track the spill. It was only last week that it announced a task force to assess the spill's actual size - a key and much-debated piece of information. There are many proposals for how it might do this (see page 421 for one example). But researchers still seem to be unclear over exactly what data NOAA is collecting.

Disasters are, by their nature, bound to be followed by disorganization and confusion. It is unrealistic to expect a 'perfect' response. And in fairness, the Gulf oil spill has been particularly difficult in this regard. Unlike earthquakes, hurricanes and most other disasters, which strike suddenly and unambiguously, the oil spill has unfolded slowly. Many days went by before it became clear just how bad the leakage was, and how big a response would be needed.

Nonetheless, aspects of the US approach could be improved. For example, the Office of Response and Restoration currently experiences a boom-and-bust funding cycle from one oil spill to the next. As the 1989 Exxon Valdez spill in Alaska's Prince William Sound has faded into memory, the office has lost around one-third of its staff, leaving the remainder stretched. One solution would be to rebuild the office and keep it at adequate staffing levels by supplementing its annual budget with money from the federal Oil Spill Liability Trust Fund, which is supported in large part by a tax on the petroleum industry and is intended to pay for the government's response to oil emergencies. This would allow for basic research into the best response efforts, along with ongoing monitoring. Another useful step would be the establishment of a cross-agency data-sharing plan for disasters, so that information would be open and publicly available, and gaps in the data would be obvious.

Meanwhile, BP, the energy company that owns the well, took a positive step of its own on 24 May when it announced that it would make up to $\$ 500$ million available over the next ten years for independent research on the spill's long-term environmental impact.

Disasters should not be viewed cold-heartedly as a chance to do some unique research, but neither should they be lost opportunities. Volcanic eruptions, hurricanes, earthquakes and oil spills push the environment to extremes, and can identify the limits of scientific knowledge. Science must not be allowed to miss out.

\section{In the public eye}

\section{Society deserves to see a return on its investment in science, but researchers need help to make their case.}

\section{T} he US National Science Foundation (NSF) is unique among the world's science-funding agencies in its insistence that every proposal, large or small, must include an activity to demonstrate the research's 'broader impacts' on science or society. This might involve the researchers giving talks at a local museum, developing new curricula or perhaps forming a start-up company.

The requirement's goal is commendable. It aims to enlist the scientific community to help show a return on society's investment in research and to bolster the public's trust in science - the latter being particularly important given the well-organized movements currently attacking concepts such as evolution and climate change.

Unfortunately, the very breadth of the requirement can leave researchers struggling (see page 416). Few of them have training in the activities involved - especially when it comes to education and outreach - and the NSF has not done enough to provide a support infrastructure to help.

Such an infrastructure does exist in embryonic form. For example, a few research institutions, including Stanford University in Palo Alto, California, and the University of Wisconsin-Madison, already have centres that aim to connect scientists with experts in teaching, education and public outreach, to equip them with the necessary skills and to disseminate best practices. And a few places, such as the University of New Mexico in Albuquerque, have developed workshops in which graduate students, postdocs and junior faculty members get professional training on how to interact with the public, media and government. Such efforts need to be expanded and institutionalized throughout the country.

Broader-impacts efforts also need to be better evaluated and rewarded. For example, the NSF should consider offering cash awards for the best broader-impact activities, the money from which could help to continue or expand the activities. This would motivate investigators to put greater effort into these endeavours, and would spread the word to other scientists about the sorts of activities that have proved successful.

Such initiatives would motivate what is really needed: a fundamental change in the culture of science to value not just achievement in the laboratory, but also work that makes science a part of people's lives.

The US Congress can help. The America COMPETES Reauthorization Act, which would extend an earlier boost given to the budgets of the NSF and two other science agencies, requires grant applicants to show that they have received support from their institutions in meeting the broader-impacts requirement. It also calls on the NSF to clarify the requirement's goals and to improve evaluation of the outreach activities. The act is being held up by political manoeuvring, despite strong bipartisan support. Congress should pass it without delay.

It is a truism to say that science and society are intertwined. But no relationship should be taken for granted. The NSF needs to help scientists show the world that their work is valuable. 